\title{
An Empirically-Calibrated Ship Domain as a Safety Criterion for Navigation in Confined Waters
}

\author{
Yueying Wang and Hoong-Chor Chin \\ (National University of Singapore) \\ (Email: sophia.wang@alumni.nus.edu.sg)
}

\begin{abstract}
There are limited studies on the use of ship domain as the safety criterion for ship navigation within confined waters. In this paper, a free-form ship domain has been developed empirically for navigation in confined waters. Two individual domains of an asymmetrical polygonal shape have been assumed around the own ship and target ship, and the size of the ship domains is assumed to be dynamically enlarged with increased ship speeds. The required safe distance modelled using the proposed ship domain takes into account dynamic changes in relative bearing and heading. The model is calibrated using the data of vessel movements in Singapore Strait and Singapore Port. An innovative process has been adopted that iteratively adjusts the parameters in the basic ship domain of a stationary ship; a speed function as well as a weight function to maximise the usage of the dataset. The resulting ship domain model compares well with existing ship domains for typical encounters, such as those of Fujii and Coldwell but represents non-typical encounters more effectively than existing models.
\end{abstract}

\section{KEY WORDS}
1. Ship Domain.
2. Safety Criterion for Navigation.
3. Empirical Model.

Submitted: 19 July 2014. Accepted: 24 June 2015. First published online: 12 August 2015.

1. INTRODUCTION. The assessment of clear space around the ship is of major importance for safe navigation. A recent research conducted by the Nautical Institute (NI) indicated that 60 per cent of collision and grounding cases are caused by direct human error (Gale and Patraiko, 2007) and the two major human related causes are "insufficient assessment of the situation" (24\%) and "poor look out" $(23 \%)$. To assess the situation, navigators often adopt a set of safety criteria such as the Distance at Closest Point of Approach (DCPA) and Time to Closest Point of Approach (TCPA) to determine if actions need to be taken. Another popular safety criterion, which was first proposed by Fujii and Tanaka (1971) is the use of ship domain - a physical spacing around the ship to ensure sufficient separation between ships. Ship domain has been adopted in collision-risk assessments (Fujii and Tanaka, 1971; Goodwin, 1975; Pietrzykowski, 2008; Pietrzykowski and Uriasz, 2009), collision-avoidance pre-emption (Dove et al., 
1986; Zhao et al., 1994), traffic simulation (Davis et al., 1980; Coldwell, 1983; Hansen et al., 2013), as well as navigational path planning (Smierzchalski and Michalewicz, 2000; Szlapczynski, 2011).

A number of shapes have been proposed to represent the ship domain. Goodwin (1975) and Davis et al. (1980) proposed circular domains while Fujii (1971), Coldwell (1983) and Kijima (2003) preferred elliptical domains and others, for example, Smierzchalski (2000) and Pietrzykowski (Pietrzykowski, 2008; Pietrzykowski and Uriasz, 2009) used polygonal domains. While regularly-shaped domains may be simple to describe and apply, they often do not adequately represent all the navigational encounters well. On the other hand, domains using complex shapes are difficult to determine, particularly if the calibration process is not rigorous.

To determine ship domains, statistical methods have been initially used, although most ship domains have not been well calibrated. The ship domains based on statistical approaches usually rely on data of ship movement trajectories (Fujii and Tanaka, 1971; Goodwin, 1975; Coldwell, 1983). However, while the trajectory data are suitable for studies of traffic capacity and channel navigation safety (Zhao et al., 1993), their use in ship domain analysis were not well established. In addition, three shortcomings of statistical methods for obtaining ship domains have been identified by Pietrzykowski and Uriasz (2009): 1) necessity for sufficient amount of data, 2) difficulties in separating the factors affecting the domain shape and size and 3) an unclear description of the perceived clear area around the ship. Therefore the statistical methods for developing ship domains still have room to improve, although Hansen et al. (2013) recently attempted to overcome these shortcomings by using a large number of observations from the Automatic Identification System (AIS).

In general, navigational waters can be divided into three categories: 1) open sea waterways, 2) confined waterways and 3) narrow fairways and channels. Most of the existing ship domains are developed for open waters (Goodwin, 1975), while very few are for narrow fairways and confined waters (Fujii and Tanaka, 1971; Pietrzykowski, 2008). The traffic densities in the three categories of waterways are significantly different. Empirically, Goodwin (1975) found that the size of ship domain is dependent on ship density and the operating environment. While there are more degrees of freedom in open oceans than in congested strait waters, it is debatable that a substantially larger ship domain is necessary for open waters or that the resulting size is limited by the lack of critical close encounters in open waters. On the other hand, in narrow fairways, the ship domain may be constrained by the fairway geometry or ship turning ability rather than just traffic influence and navigational behaviour (Pietrzykowski, 2008). Consequently, the trajectory data may be rather biased by fairway geometry to enable a fair representation of the ship domain. A study on ship domain using data in confined waters is most promising but there are only a few studies available, e.g., Hansen et al. (2013).

Navigation within confined waters is influenced not just by traffic density in the area but also the presence of general as well as locally-imposed traffic rules. One of the principal rules in navigation is the International Regulations for Preventing Collisions at Sea 1972 (COLREGS, 1972), which stipulate give-way and stand-on behaviours according to specific ship encounters. For most ship domain models, the definitions of ship encounter are crude. For instance, they are usually grouped into three broad encounters, i.e., overtaking (or being overtaken), head-on and crossing, according to the relative heading and bearing. This results in an undesirable abrupt change in 
safety criterion at the interface between two encounter types due to a change in relative heading and bearing.

To overcome the above limitations, a rigorous approach to modelling ship domains in confined waters is proposed. This paper has developed a free-form ship domain for risk assessment in confined waters. The methodology for developing and evaluating the model is described in Section 2. The calibrated model of ship domain is presented in Section 3, which is followed by a discussion of the proposed model in Section 4. Section 5 summarises the key findings of this research and highlights the potential areas for future work.

\section{METHODOLOGY}

2.1. Formulation of Ship Domain. To take into account attributes of both ships in an encounter, the safe spacing that the ships will keep from each other is modelled to comprise two components; one belonging to the Own Ship (OS) and the other Target Ship (TS). Further, since the safe spacing is also influenced by the heading of the two ships, we then formulate the concept of two individual ship domains around the ship, the size of which is dependent on the Length Overall (LOA) and the current speed. The safe distance which both ships will keep from each other is considered as the sum of the length of each of the domains (i.e., $\mathrm{SD}_{\mathrm{OS}}$ and $\mathrm{SD}_{\mathrm{TS}}$ for $\mathrm{OS}$ and $\mathrm{TS}$ respectively shown in Figure 1) in the direction of the line of sight between the ships. Conceptually, it is as though ships will navigate to ensure the two individual domains will not encroach into each other. We consider the two individual ship domains to assume a unified domain model determined by its ship attributes and calibrated using observed navigational data.

The proposed perspective should effectively represent the safe navigational water between approaching ships, and reflect the navigational features of both ships and their interactive navigational behaviour. Instead of adopting a simplified and restricted elliptical domain around each ship, this study assumes an asymmetrical polygonal shape with small discretized intervals, thus offering it a higher degree of freedom. In addition to relaxing the limitation on the shape, the size of the ship domain will be dynamically enlarged with increasing ship speed based on a consistent basis of the domain. Furthermore, the required safe distance between ships governed by the edge of the ship domain will also change with changing relative bearing and heading throughout the encounter. Based on this concept, the domain model will include three components: the representation of domain shape, the representation of the basic domain size for the stationary ship and finally the effect of the ship speed function.

The individual domain is defined in the shape of an asymmetrical polygon with $n$ number of vertices and the boundary of the domain is formed by joining the $n$ vertices sequentially. The size of the polygon is measured by the radial distance $R$ from the ship centre to the different vertices of the polygon, defined by a polar angle $\theta_{i}$ clockwise from the ship heading and it is governed by a function of ship LOA $(L)$ and speed $(v)$ :

$$
R_{\theta_{i}}=\alpha_{\theta_{i}} \cdot L \cdot g_{\theta_{i}}(v)
$$

where $i(i=1, \ldots, n)$ is the indicator of vertex, and $n$ is the total number of vertices based on specified angular interval discretization $\Delta$ (in degrees) such that $n=360 / \Delta$; $\alpha_{\theta_{i}}$ is the normalised radial distance of the domain when the vessel is stationary at the polar angle $\theta_{i} ; g_{\theta_{i}}(v)$ is a speed function which governs how the domain is expanded 


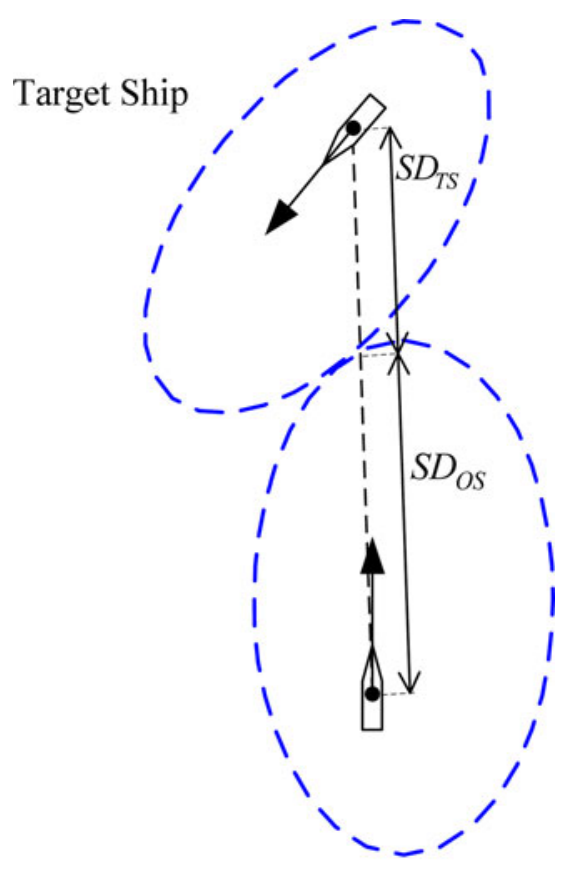

Own Ship

Figure 1. Definition of safe spacing.

with non-zero value of $v$ at the polar angle $\theta_{i}$. This formulation is shown graphically in Figure 2. The vector $\alpha_{\theta_{i}}$ representing the normalised zero-speed domain is to be calibrated along with parameters defined in the speed function. Note that as a normalised vector, $\alpha_{\theta_{i}}$ explains the shape of the ship domain and by assuming that the ship domain is proportional to the LOA, the size of the zero-speed domain can also be determined. It is further assumed that while $\alpha_{\theta_{i}}$ is assumed to be well defined and invariant to other factors, a different vector may be derived for different environmental conditions. For example, a different set of $\alpha_{\theta_{i}}$ values will be obtained for day and night conditions.

The speed function $g_{\theta_{i}}(v)$ serves as a size adjustment for scaling up the zero-speed ship domain. The speed functions are specified for four axial directions and the speed component for other directions are interpolated based on the calibrated speed functions in the axial directions. Suppose that the speed functions in the four axial directions, i.e., fore, aft, port and starboard sides are defined as $g_{f}(v), g_{a}(v), g_{p}(v)$, $g_{s}(v)$ respectively. The effect of speed on ship domain is generally non-linear (Smierzchalski, 2000; Kijima et al., 2006), with the domain size increasing with speed initially but tapering off at higher speeds. Therefore, a suitable formulation of the speed function might be a quadratic function defined as

$$
g(v)=1+\lambda v+\mu v^{2}
$$

in which $\lambda$ and $\mu$ are the parameters to be determined. Allowing different speed functions for the four axial axes will result in eight degrees of freedom, i.e., eight calibration parameters $\lambda_{f}, \mu_{f}, \lambda_{a}, \mu_{a}, \lambda_{p}, \mu_{p}, \lambda_{s}, \mu_{s}$. 


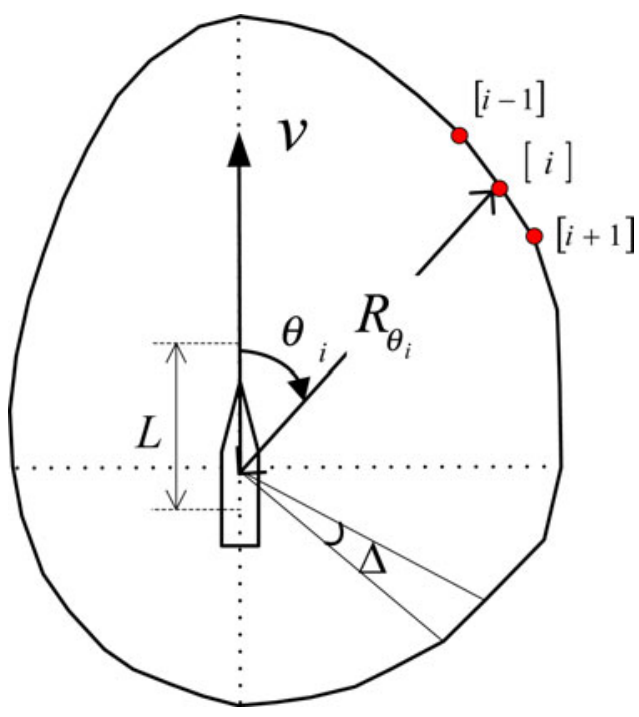

Figure 2. Representation of ship domain.

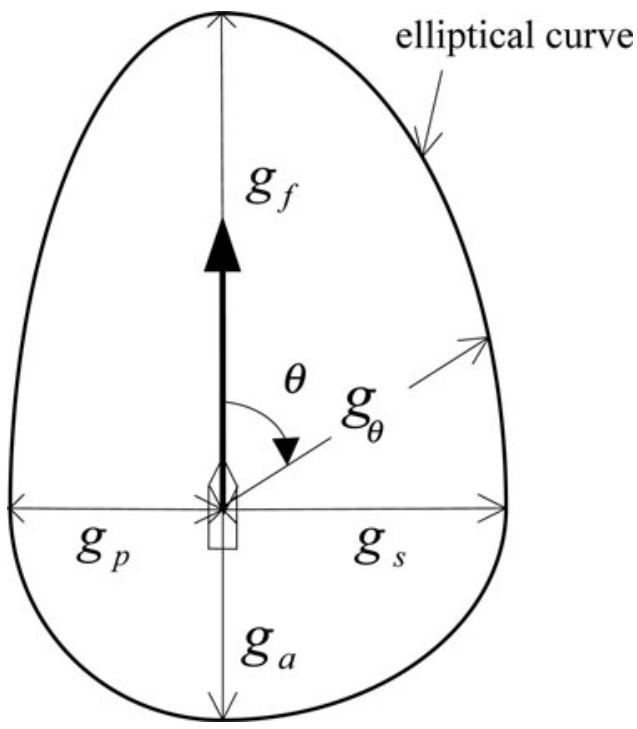

Figure 3. Illustration of four directions of speed functions.

Further, assuming the speed function is governed by an elliptical form as seen in Figure 3 , the speed in any given heading $\theta$, i.e., $g_{\theta}(v)$ and in short, $g_{\theta}$ will be

$$
g_{\theta}=\frac{\left[(1+m) g_{f}+(1-m) g_{a}\right] \cdot\left[(1+n) g_{s}+(1-n) g_{p}\right]}{2 \sqrt{\left[(1+m) g_{f} \sin \theta+(1-m) g_{a} \sin \theta\right]^{2}+\left[(1+n) g_{s} \cos \theta+(1-n) g_{p} \cos \theta\right]^{2}}}
$$


where $m$ and $n$ are defined as

$$
\begin{gathered}
m=\left\{\begin{aligned}
1, & \theta \in[-\pi / 2, \pi / 2) \\
-1, & \theta \in[-\pi,-\pi / 2) \cup[\pi / 2, \pi)
\end{aligned}\right. \\
n=\left\{\begin{aligned}
1, & \theta \in[0, \pi) \\
-1, & \theta \in[-\pi, 0)
\end{aligned}\right.
\end{gathered}
$$

\subsection{Calibration of Ship Domain Model}

2.2.1. Iterative Calibration Process. Due to the high degree of inter-relationships between the ship domain model and the speed function as well as the statistical uncertainties inherent in the data, it is difficult to derive a close-form solution of the parameter set. To determine the parameter values of the zero-speed domain model and the speed function, an iterative calibration procedure is adopted. The structure of the procedure is given in a flow chart shown in Figure 4. For the two sets of interrelated parameters in the ship domain model, at least one set of parameters needs to be assumed to start the interactive process. For convenience, taking the idea of the swinging circle of a ship anchoring position, a circular zero-speed domain of size $L$, i.e., $\alpha_{\theta}=1, \forall_{\mathrm{i}}$ is initially assumed. Then the parameters in the speed function are determined, followed by an estimation of the weight function and a re-computation of the zero-speed domain. Since it is a free-form polygonal shape, non-convexity may exist at the boundary of the ship domain. In order to avoid potential fluctuation in using the ship domain, a step of smoothing the boundary of the ship domain is developed to ensure the zerospeed domain is convex. With the re-computed ship domain model, each ship-pair encounter needs to be reassessed to ascertain how likely the ship pair is to have a close encounter, resulting in possibly a new set of parameter values of the speed function as well as the ship domain. This procedure is iterated until convergence in the parameter values is achieved.

2.2.2. Method for Estimating Parameters. The calibration procedure is set as an optimisation problem, with the parameters estimated by optimising the distance between the model behaviour and the extracted close encounters data. This is formulated as

$$
\begin{array}{cc}
\text { Minimize } & F n\left(\alpha_{k}, \lambda_{x}, \mu_{x}\right) \\
\text { Subject to } & \\
& \alpha_{k}>0 \\
& \lambda_{x}+2 \mu_{x} v_{\max }>0 \\
\lambda_{x}>0 \\
\mu_{x}>0
\end{array}
$$

where $F n$ stands for the objective function of the optimisation problem involving two sets of parameters; $\alpha_{k}(k=1,2, \ldots, 2 \pi / \Delta)$ are a set of parameters governing the zerospeed domain, and $\Delta$ is the angular discretization interval; $\lambda_{x}$ and $\mu_{x}$ are the parameters in the speed function in which $x=f, a, p, s$ representing the fore, aft, port and starboard side; $v_{\text {max }}$ is the maximum achievable speed. While the speed function is assumed to follow the quadratic form, it is necessary to constrain the parameters to the 


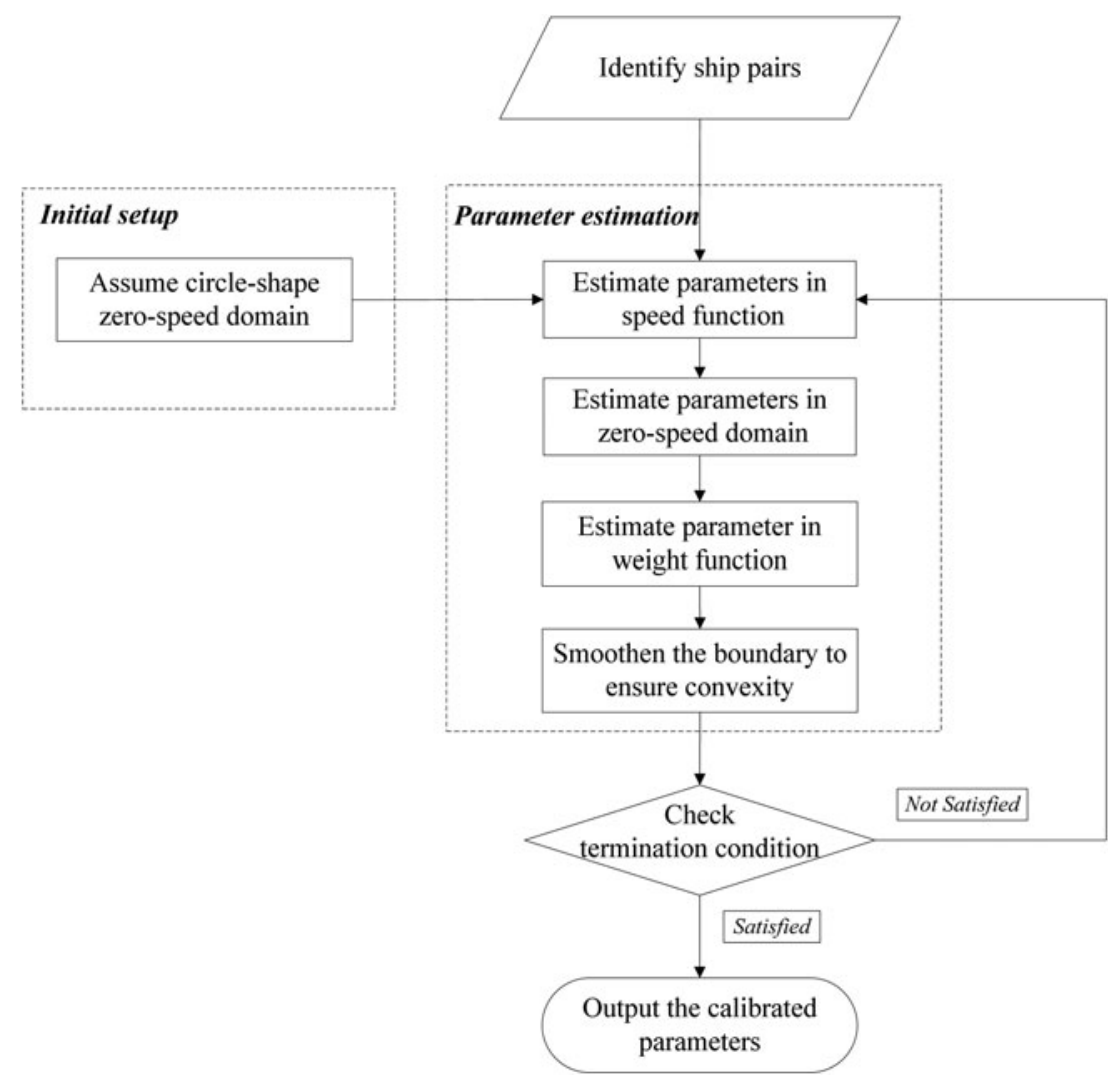

Figure 4. Flow chart showing calibration procedure of ship domain model.

maximum $v_{\max }$ so that only the monotonically increasing portion of the quadratic curve is used.

The optimisation function essentially seeks to minimise the errors between the safe distances obtained from the ship domain models and the observed values. The relative error function adopted is given by

$$
E_{r e l}=\frac{|E|}{d}=\frac{\left|d-S D_{O S}-S D_{T S}\right|}{d}
$$

where $E$ is the estimation error, $d$ is the observed distance between the two ships and $S D_{O S}$ and $S D_{T S}$ are the lengths of the domains in the direction of line of sight based on the relative bearing and heading of the two ships.

Among all the ship pairs, it is not known, a priori whether a particular pair in close range is necessarily in close encounter. Including all possible data pairs would make the calibration procedure tedious and superfluous, so some form of data reduction is necessary. In general, ships nearer the subject ship, i.e., OS, are more likely to be in close encounter and hence there is a higher probability that the space separation between the two ships will be governed by the ship domains to be modelled. This probability will decrease with larger space separation, vanishing quickly with the distance apart. 
Therefore, a weight function $(w(E))$ is defined in Equation (8) to account for the contribution of each ship pair data, and favour the ship pairs in close encounters. Since a larger $E$ may be a result of lower likelihood of the ships being in close encounter, a modified exponential function is applied as

$$
w(E)= \begin{cases}e^{-\omega E}, & E \geq 0 \\ 1, & E<0\end{cases}
$$

in which $\omega$ is the parameter of the exponential function. The objective function to be minimised is thus defined as the sum of the weighted relative error for each encounter, i.e.,

$$
F n=\sum E_{r e l} \cdot w(E)
$$

The optimisation problem is solved using the Genetic Algorithm (GA) technique. The implementation of GA in this research is coded under the programming environment of MATLAB. The Global Optimisation toolbox in MATLAB provides various optimisation techniques including GA and it supports algorithmic customisation for users' purposes. For instance, the user can create a custom generic algorithm variant by modifying initial population and fitness scaling options or by defining parent selection, crossover, and mutation operators (MathWorks, 2012).

\section{DATASET}

3.1. Background. For the purposes of this paper, vessel movement data in Singapore Port and Singapore Strait were obtained from the Vessel Traffic Information System (VTIS) database operated by the Maritime and Port Authority of Singapore (MPA). A chart of Singapore VTIS coverage (known as STRAITREP operation areas) from the MPA website is shown in Figure 5 including Singapore Strait (Sector 7, Sector 8 and Sector 9) and Singapore Port. Seven hours of vessel movements covering both day and night conditions, at scan intervals of every 2 seconds were used. The data include the coordinate position, recorded speed and heading of each vessel tracked as well as the ship attributes, i.e., ship LOA, height, draft, Gross Tonnage (GT) and the Maritime Mobile Service Identity (MMSI) number.

3.2. Data Preparation. To extract only relevant and useful information for analysis, some vessels captured under the VTIS were excluded. These include vessels that are anchored or moored, ships under special missions that may not follow the typical navigational rules, such as tug boats, police patrols and bunkering vessels as well as ships without an MMSI number such as fishing vessels and yachts.

Following the data reduction process, a total of 624 vessels were captured during the survey period. Of these about $66 \%$ of the ships have LOA ranging from $50 \mathrm{~m}$ to $200 \mathrm{~m}$ with the longest ship up to $400 \mathrm{~m}$. Tracking their positions throughout their movement within the study area, a total of around 2.7 million ship positions were obtained. Of these $57 \%$ are recorded in day time conditions (from 10:00 AM to 2:00 PM). The distribution of speeds of these vessels throughout their movement in the port is plotted in Figure 6. The wide variation of speeds should allow a good calibration study of the navigational behaviour. Based on the ship positions, 264,975 ship pairs have been identified for day time condition within a circular range with $2 \mathrm{~nm}$ radius. These ship pairs will seed the process of domain calibration with an initial dataset as indicated in Figure 4. 


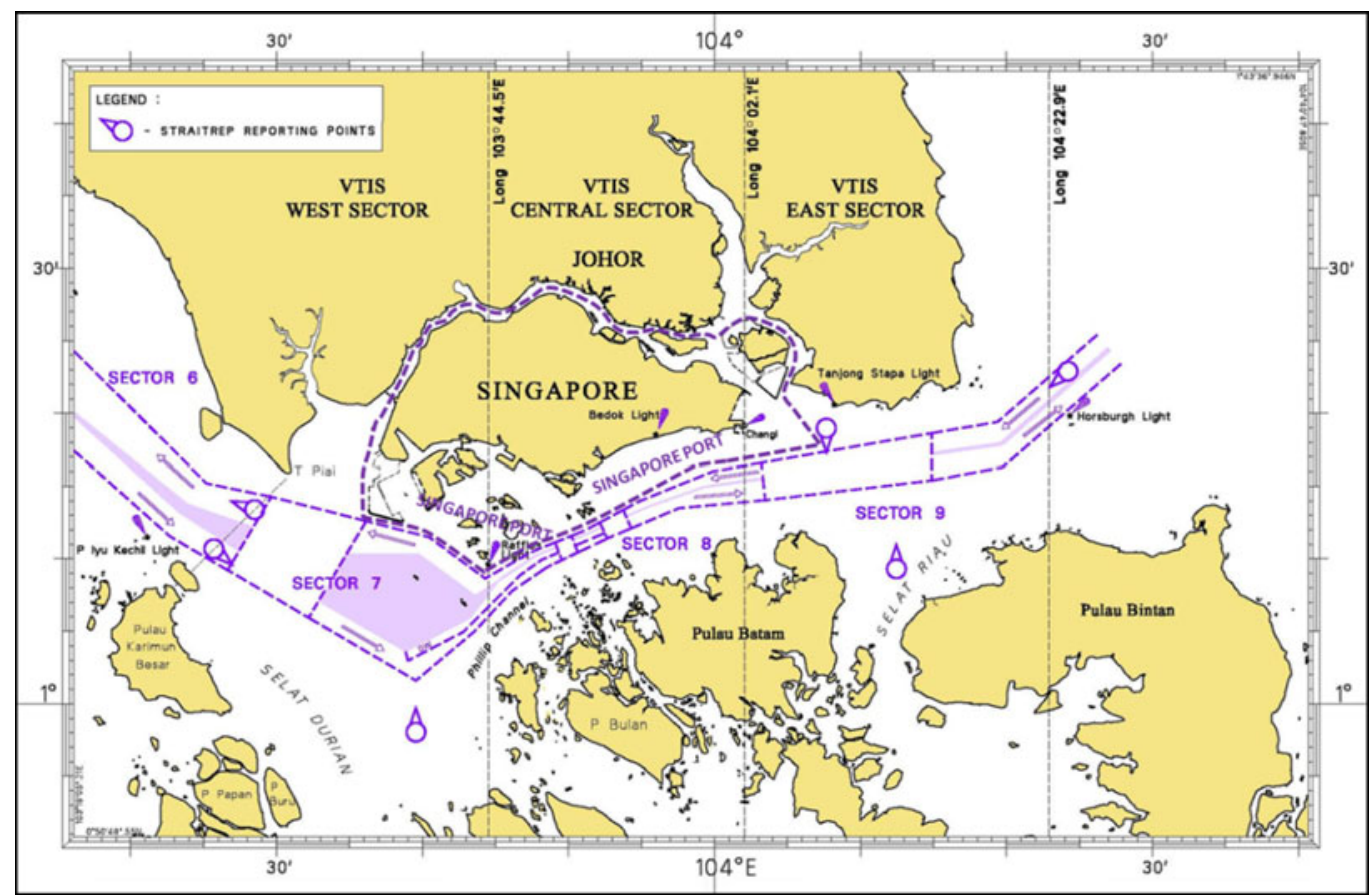

Figure 5. Map of Singapore VTIS coverage including Singapore Strait and Port. 


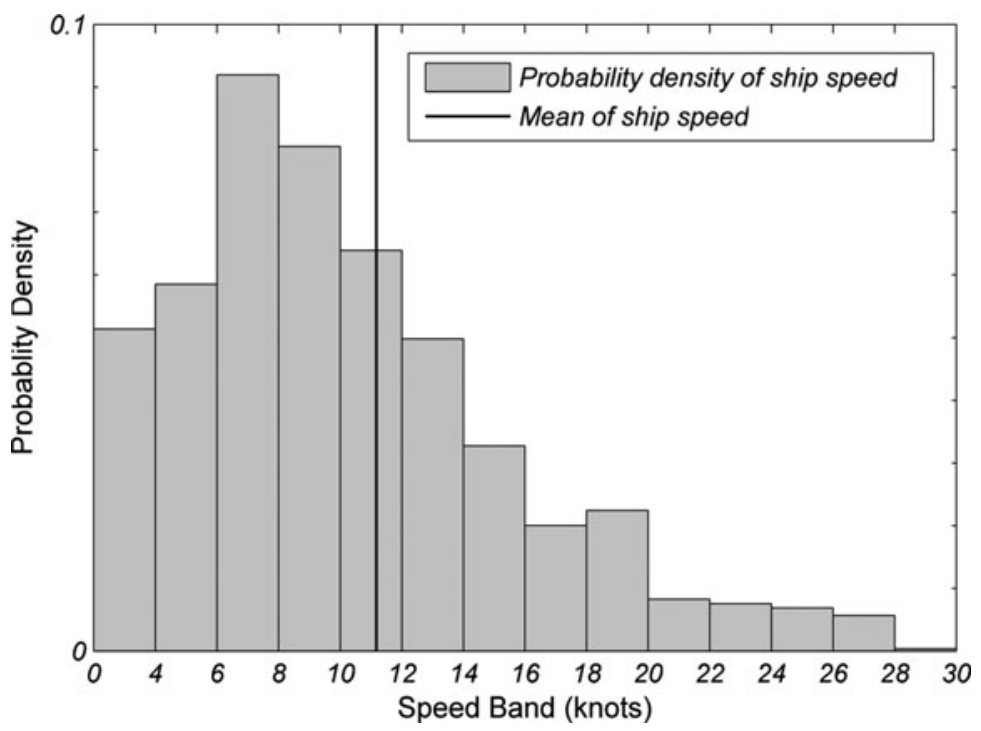

Figure 6. Probability density plot of ship speed.

4. RESULTS OF CALIBRATING SHIP DOMAIN MODEL. Normalising the ship domains with the ship LOA and at zero-speed, we obtain a calibrated nondimensional ship domain which is independent of the LOA as well as ship speed as shown in Figure 7 along with the calibrated speed functions in Figure 8 for the four major sides, i.e., the fore, aft, port and starboard sides as illustrated in Figure 3 which will be used to scale up the normalised ship domain.

From Figure 7, the zero-speed domain is relatively circular in shape although the fore side of the zero-speed domain is obviously larger than the aft side, the starboard side and port side. Figure 8 shows that the domain enlarges significantly on the fore and aft sides with increasing speed while it enlarges marginally on the port and starboard sides. The value of the speed function on the fore side is consistently higher than that on the aft side at all speeds and it reaches $7 \cdot 2$ at 30 knots on the fore side compared 5.9 on the aft side. The values of speed function on the port and starboard sides are around 3.0 at the speed of 30 knots though it is slightly higher on the starboard side than that on the port side for all speeds. The enlargement of ship domain due to increased ship speed is shown in Figure 7 where the non-dimensional ship domains at the speeds of 0 knots and 15 knots are depicted. These results suggest that navigators are comparatively more sensitive to ships from the fore and aft directions than those approaching from the port and starboard sides, and more sensitive to ships in front than those going after it. It may be also inferred that the own ship is more likely to give way to ships from the starboard sides than those coming from the port sides.

5. DISCUSSION. This section aims to examine the usefulness of the proposed ship domain model in this paper to model safe spacing between moving ships. It is first achieved by evaluating the fitness of ship domains in representing real navigational 


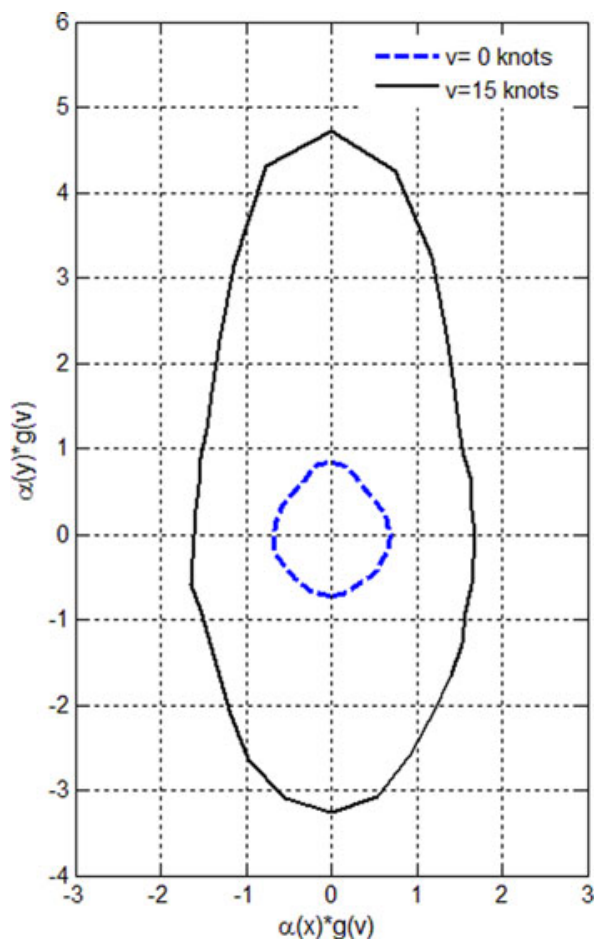

Figure 7. Non-dimensional ship domains at zero speed and 15 knots.

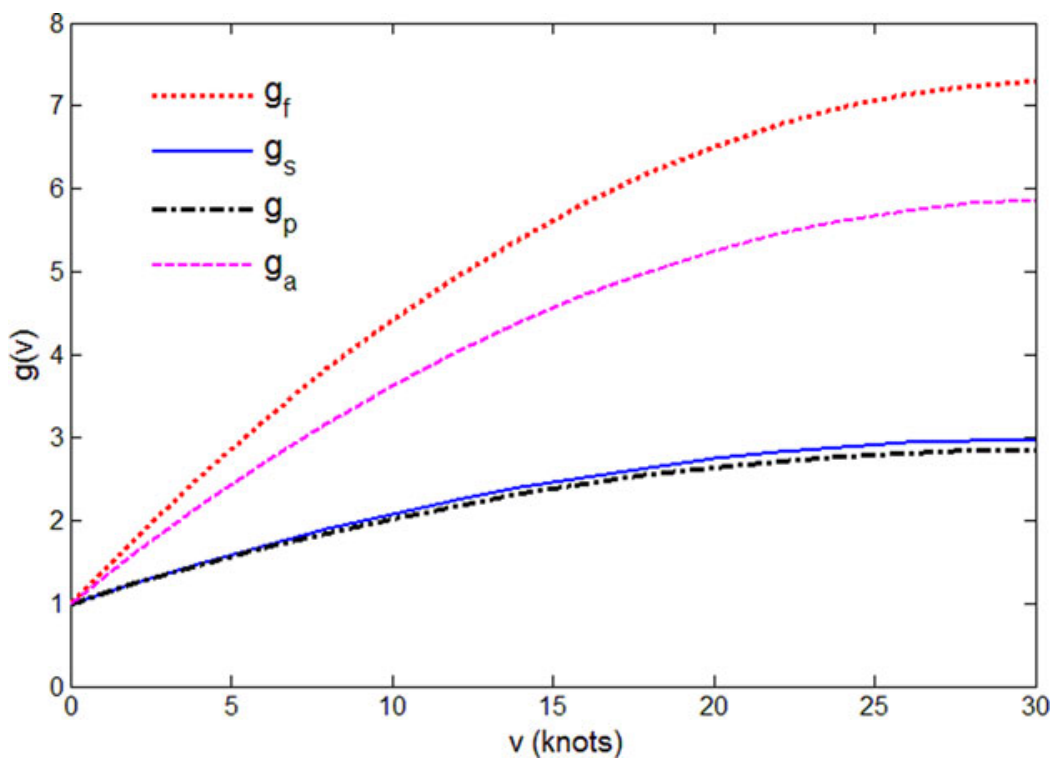

Figure 8. Speed functions at the four sides. 
behaviour inferred from traffic movement data. The proposed model in this paper and selected existing models of ship domain are considered. Using several case scenarios, the safe spacing developed in the proposed model in this paper is further compared with that derived from domain models in some earlier studies. This involves a systematic comparison of the shape and size taking into account factors such as ship heading and relative bearing, as well as whether the domain is around OS or TS.

5.1. Comparison of Models of Ship Domain Based on Proportion of Encroachment. There are a number of empirically-derived ship domains that have been previously studied. For the purpose of this comparison, two elliptical domains invariant with ship speeds developed by Fujii and Tanaka (1971) and Coldwell (1983) will be used for the comparison with our proposed model. Based on data of overtaking encounters in Japanese waters, Fujii and Tanaka (1971) assumed the magnitude of major and minor semi-axis of the ellipse ( $r$ and $s$ respectively) to be proportional to the ship length, i.e., $r=7 L$ and $s=3 L$. On the other hand, Coldwell (1983) found $r=6$ cables and $s=1.75$ cables.

The comparison of the different safe spacing kept is made on the basis of the amount of encroachment observed from the VTIS data. Encroachments are instances in which ships are in closer proximity than predicted by ship domain. If the ship domain is assumed correct, i.e., the critical encounter is when $\mathrm{d}=\mathrm{SD}$, then the number of encroachments, i.e., when $\mathrm{d}<\mathrm{SD}$, possibly represents the error associated with overestimating the ship domain. On the other hand, if a deterministic ship domain is assumed, then ships found to lie beyond the predicted ship domain, i.e., $\mathrm{d} \gtrsim$ SD may represent some form of under-estimation, although it may be argued that ships are not always at the critical position. Hence the true domain will be in a region where the error of over-estimation is minimal and where the error of under-estimation is rapidly rising.

The errors of under-estimation or over-estimation of the safe spacing between ships modelled by the proposed ship models are compared with those generated by Fujii's models and Coldwell's models. The ratio of the actual distance between the two ships to the required safe distance, denoted by d/SD is used for evaluation. The percentages of encounters with respect to increment values of d/SD for three different domain models are plotted in Figure 9.

A low percentage of encroaching encounters, i.e., where $\mathrm{d}<\mathrm{SD}$, implies the chance that the ship domain is less likely to be smaller than predicted while a high percentage of encounters falling just beyond the predicted domain represent a strong resistance for the ship domain to be larger. Figure 9 shows that Coldwell's model has been overestimated since the percentage of encounters below the predicted domain $(\mathrm{d} / \mathrm{SD}<1)$ is high. Clearly, of the three models, Coldwell has a larger and hence over-estimated domain. Compared to Fujii's domain, the proposed domain is slightly better in yielding a slightly smaller error of over-estimation. On the other hand, the proposed domain gives a higher percentage of encounters just beyond the predicted domain than Fujii's model, suggesting that it has a higher resistance to under-estimation than Fujii's model. Taken together, it may be said that the proposed model gives the best solution.

5.2. Comparison of Ship Domains using Case Studies. This section compares the safe distances from the proposed model of ship domain with those generated from selected earlier studies under a variety of scenarios. Most previous models employ a single domain to represent the safe distance between two ships whereas the proposed model employs two individual domains around both OS and TS; an equivalent single 


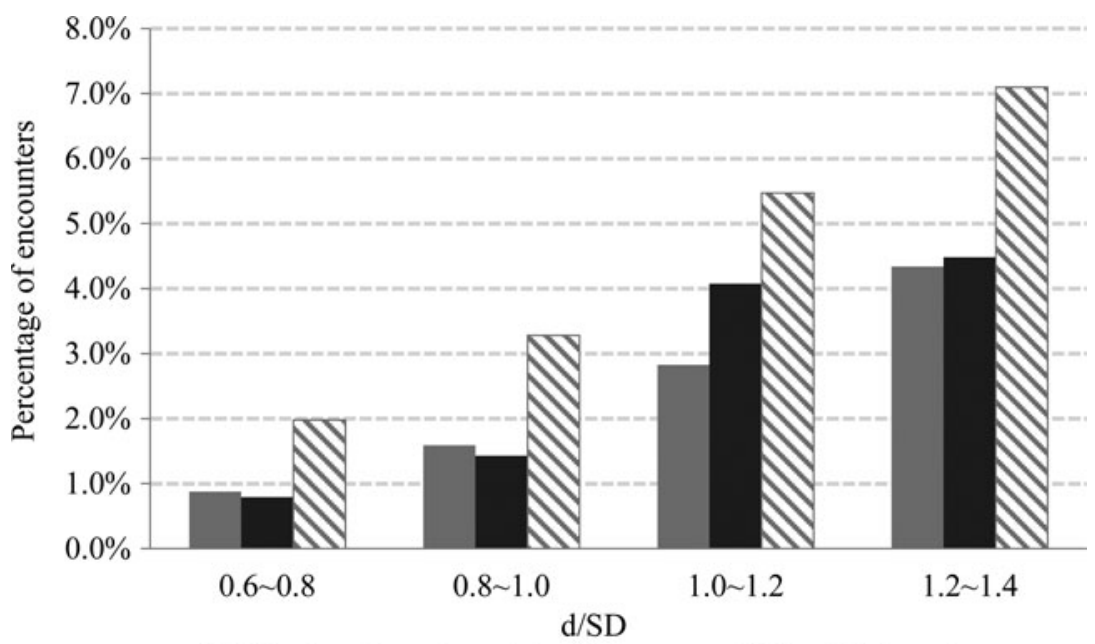

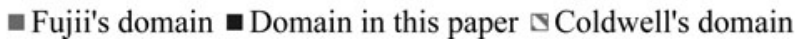

Figure 9. Percentage of encounters in the vicinity of critical encounter.

Table 1. Ship attributes of OS and TS in comparison with Goodwin's model.

\begin{tabular}{lcrr}
\hline & OS (1) & OS (2) & TS \\
\hline LOA(m) & 200 & 250 & 200 \\
Speed(knots) & 15 & 20 & 0 \\
Heading(degree) & 0 & 0 & - \\
\hline
\end{tabular}

Note: TS is stationary therefore no heading for TS.

domain is used to represent the safe distance from the proposed model. Some models only consider ship LOA and/or speed of a single ship, either OS or TS; the proposed model in this paper considers attributes of both OS and TS, a scenario involving a moving ship and a stationary ship is used to ensure compatibility in comparison.

5.2.1. Comparison with Circular-Type Domain. The safe distance from the proposed ship model is first compared with that of the circular-type model developed by Goodwin (1975) which has three unequal sectors with different radii, but are invariant with the ship speed and length. Since Goodwin's model is developed for single ship, a basic situation of a moving OS and stationary TS is examined under two scenarios: (1) a smaller and slower OS and (2) a larger and faster OS shown in Table 1.

As shown in Figure 10, the safe distance based on the proposed model of a moving OS and stationary TS is superimposed on Goodwin's model for the two scenarios. There are clearly distinctive differences between the proposed model and Goodwin's model. Goodwin's model oversimplifies the safe spacing with large discontinuities at the sector boundaries. In addition, compared to the proposed model, Goodwin's model overestimates the safe distance, particularly on the port and starboard sides. This is partly because Goodwin's model is suitable for open seas (Zhao et al., 1993). There is however, closer similarity along the longitudinal axis. Nevertheless, since Goodwin's model does not account for the size of the ships and the speeds, there is 


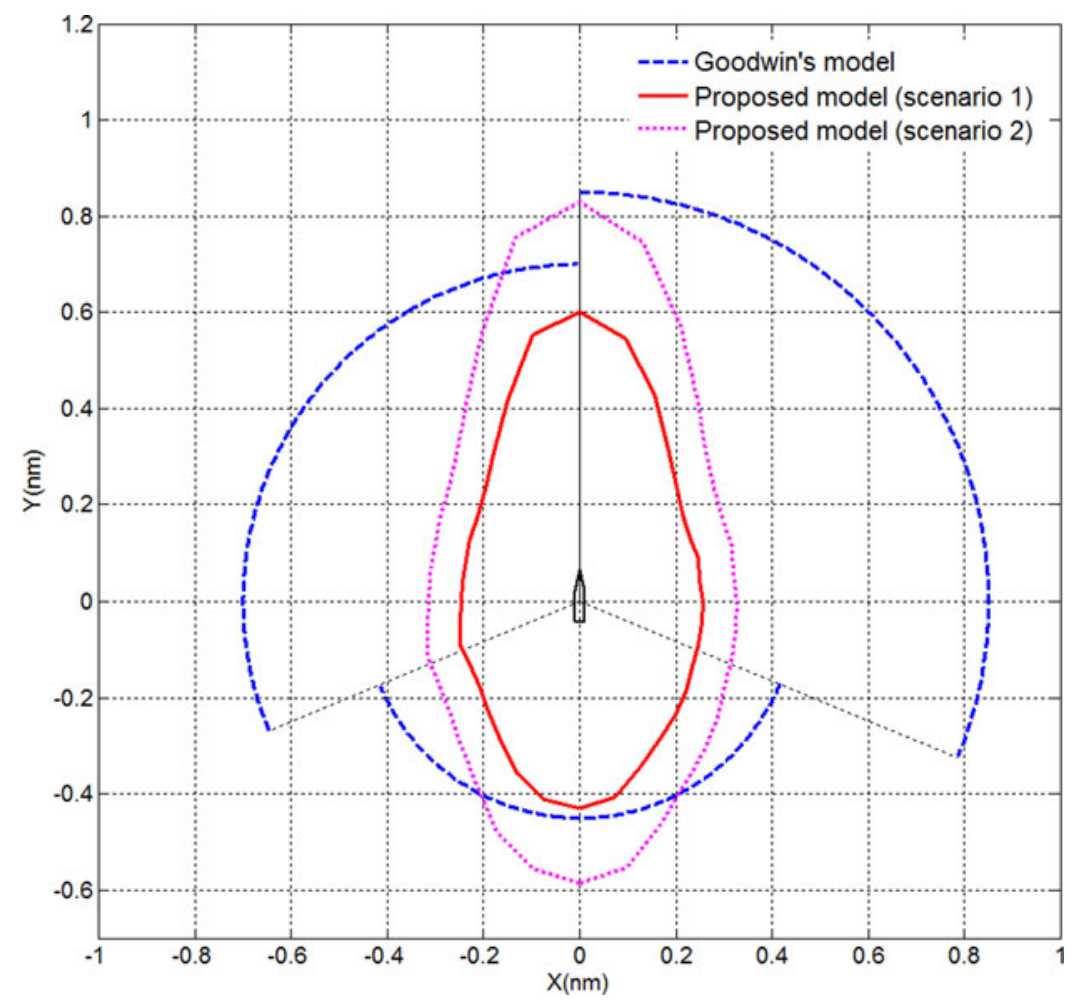

Figure 10. Comparison of safe spacing between proposed model and Goodwin's models.

Table 2. Ship attributes of OS and TS in comparison with Coldwell's model.

\begin{tabular}{lrrr}
\hline & OS $(1)$ & OS $(2)$ & TS \\
\hline LOA(m) & 200 & 200 & 200 \\
Speed(knots) & 15 & 20 & 0 \\
Heading(degree) & 0 & 0 & - \\
\hline
\end{tabular}

a closer match between the proposed model and Goodwin's model on the aft side for the smaller and slower OS and on the fore side for the larger and faster OS. It may be argued that these are closer to the critical conditions in less restricted waters.

Compared to Goodwin's model, the proposed model may be advantageous since it takes into account the ship size and dynamic effects. In addition, the comparison also implies that Goodwin's model is suitable for large and fast vessels because the vessels observed by Goodwin were large and medium ships (Zhao et al., 1993).

5.2.2. Comparison with Elliptical-Type Domains. Two elliptical-type domains are evaluated in this section-Coldwell's model (Coldwell, 1983) which is developed around the OS and Fujii's model which is built around the TS. Both of the models are dependent on the ship LOA but independent of its speed.

Comparison with Coldwell's model is based on two scenarios representing two speed conditions of a large OS and a stationary TS as shown in Table 2. Figure 11 shows the 


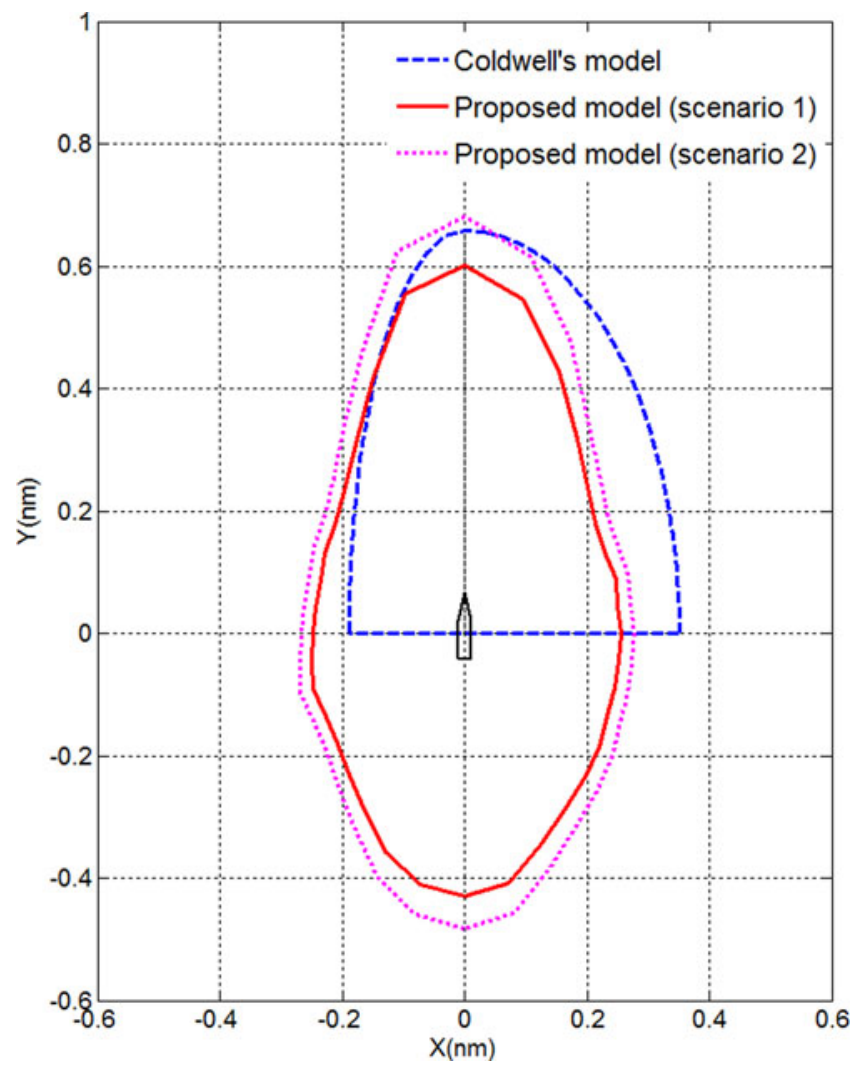

Figure 11. Comparison of safe spacing between proposed model and Coldwell's model.

comparison between Coldwell's model and the proposed model for the two scenarios in a head-on encounter of OS with LOA $=200 \mathrm{~m}$ and speeds of 15 and 20 knots. The comparison shows that the proposed model is reasonably compatible with Coldwell's model on the fore side but rather different on the starboard and port side. It should be noted that to account for the influence of general navigational rules on navigators' behaviour, i.e., to pass on the port side instead of the starboard side in head-on encounter, the safe spacing of Coldwell's model on the starboard side is larger than that on the port side. However, the preference to pass on the port side may not be equated to navigators having a lower tolerance of separation on the port side than the starboard side, especially in the empirical domain model developed from real navigational data.

The comparison with Fujii's model (Fujii and Tanaka, 1971) is based on three scenarios with a moving OS of LOA $=100 \mathrm{~m}$ at speeds of 10 knots and 15 knots and a stationary TS of LOA $=100 \mathrm{~m}$ as tabulated in Table 3 . The result of safe spacing from the two models is shown in Figure 12 and it can be seen that the proposed model with the same ship dimensions of OS and TS in scenario 1 approximately matches with Fujii's model although predicting slightly larger lateral sides and fore side and slightly smaller aft side. Furthermore, with a faster OS in scenario 2, the proposed model has been 
Table 3. Ship attributes of OS and TS in comparison with Fujii's model.

\begin{tabular}{lcrr}
\hline & OS (1) & OS (2) & TS \\
\hline LOA(m) & 100 & 100 & 100 \\
Speed(knots) & 10 & 15 & 0 \\
Heading(degree) & 0 & 0 & - \\
\hline
\end{tabular}

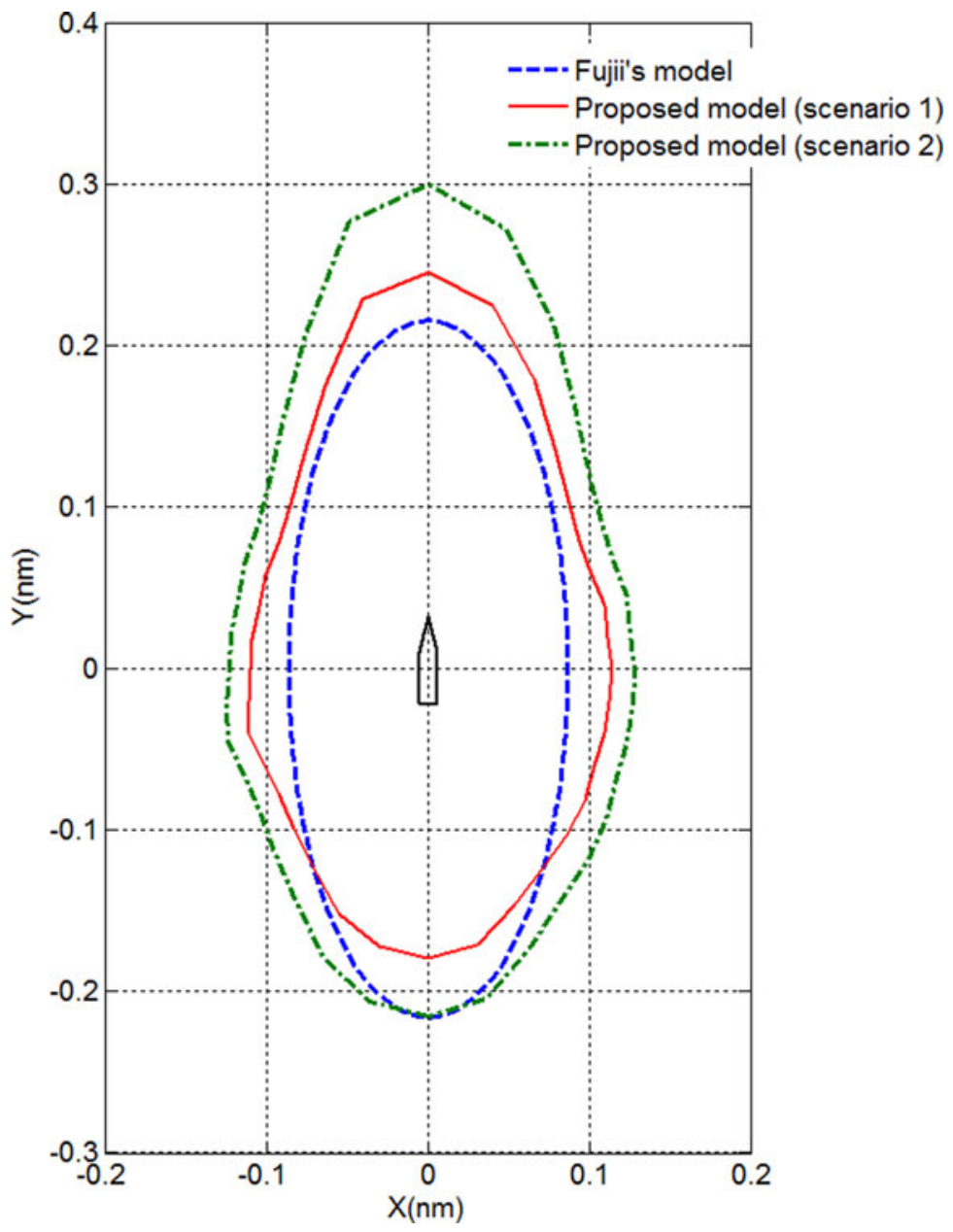

Figure 12. Comparison of safe spacing between Fujii's model and proposed model.

enlarged substantially on the fore and aft sides; it then has matched quite well with Fujii's model on the aft side.

Clearly the proposed model has a higher degree of freedom in the domain shape, e.g., by allowing different sizes on the fore and aft side, compared to the symmetrical elliptical domain which is constrained by the axial diameters. Consequently, the calibrated free-form domain model may give a better representation of reality than the 
Table 4. Ship attributes of two moving ships in comparison with Pietrzykowski's model.

\begin{tabular}{lrrrrr}
\hline Encounter & \multicolumn{2}{c}{ Head-on } & & \multicolumn{2}{c}{ Crossing } \\
\cline { 2 - 5 } \cline { 3 - 6 } Ship & \multicolumn{1}{c}{ OS } & TS & OS & TS \\
\hline LOA(m) & 300 & 300 & 300 & 300 \\
Speed(knots) & 15 & 15 & 15 & 15 \\
Heading(degree) & 0 & 180 & 0 & 225 \\
\hline
\end{tabular}

constrained elliptical domain model. Moreover, the enlarged domain due to faster ship speed in the proposed model also suggests that Fujii's model, unlike the proposed model, is unable to account for the effect of the ship speed. Quite naturally, with a higher speed, the OS will require a larger separation from the TS and vice versa for a lower speed. This highlights the superiority of the proposed model that is sensitive to the ship speed.

5.2.3. Comparison with Polygonal-Type Domains. Based on the input from experienced navigators in a desktop calibration, Pietrzykowski and Uriasz (2009) developed a polygonal model suitable in open sea for a specific pair of moving ships, in which both the ship length and speeds are considered.

Pietrzykowski's model uses a 24-sided polygon and a fuzzy function to reflect the level of navigational safety, denoting this by $\gamma \in[0,1]$ where $\gamma=0$ represents the very safe situation and $\gamma=1$ represents the very dangerous situation (collision). For the purpose of comparison, two specific encounters, i.e., a head-on encounter and a crossing encounter with the ship attributes shown in Table 4 are considered. The safe spacing generated by Pietrzykowski's model with three levels of danger represented by $\gamma=0.5,0.8,0.9$, along with the predicted safe spacing of the proposed model are shown in Figure 13 and Figure 14 for the two encounters respectively.

The comparison shows that the safe spacing in Pietrzykowski's model for both the head-on and crossing encounters, under the dangerous situation with $\gamma=0.9$ prior to any collision is much smaller than that of the proposed model, though the shape of the two models are about similar. The proposed model appears to match Pietrzykowski's model for the case with $\gamma=0.8$ particularly on the fore side, the port and starboard sides. Thus it is very reasonable to assume that Pietrzykowski's model for open seas under a relatively critical condition (assumed to be $\gamma=0.8$ ) may be close to the case of ship movements in restricted waters. It should be noted that for the aft side in both encounters, the proposed model is significantly larger than that of Pietrzykowski's model for $\gamma=0.8$ and even for a more favourable condition of $\gamma$ $=0 \cdot 5$. This is reasonable and may be attributable to the way the aft side of the domain is obtained in the two models. Pietrzykowski derived the model from navigators in a desktop exercise assuming a hypothetically static situation exists, while in fact, under a dynamic situation, such a critical situation may not be possible. For example, while it may be possible to put a limiting comfortable space separation between two ships that are moving away from each other in opposite directions, such a critical situation is practically impossible and hence never observed. As the proposed model is calibrated using actual encounters rather than a desktop input, the lower likelihood of such critical encounters will also mean a lack of empirical data to justify a smaller domain under this situation. This may also explain why most empirical models (Coldwell, 1983; Fujii and Tanaka, 1971) as shown in Figure 11 and 


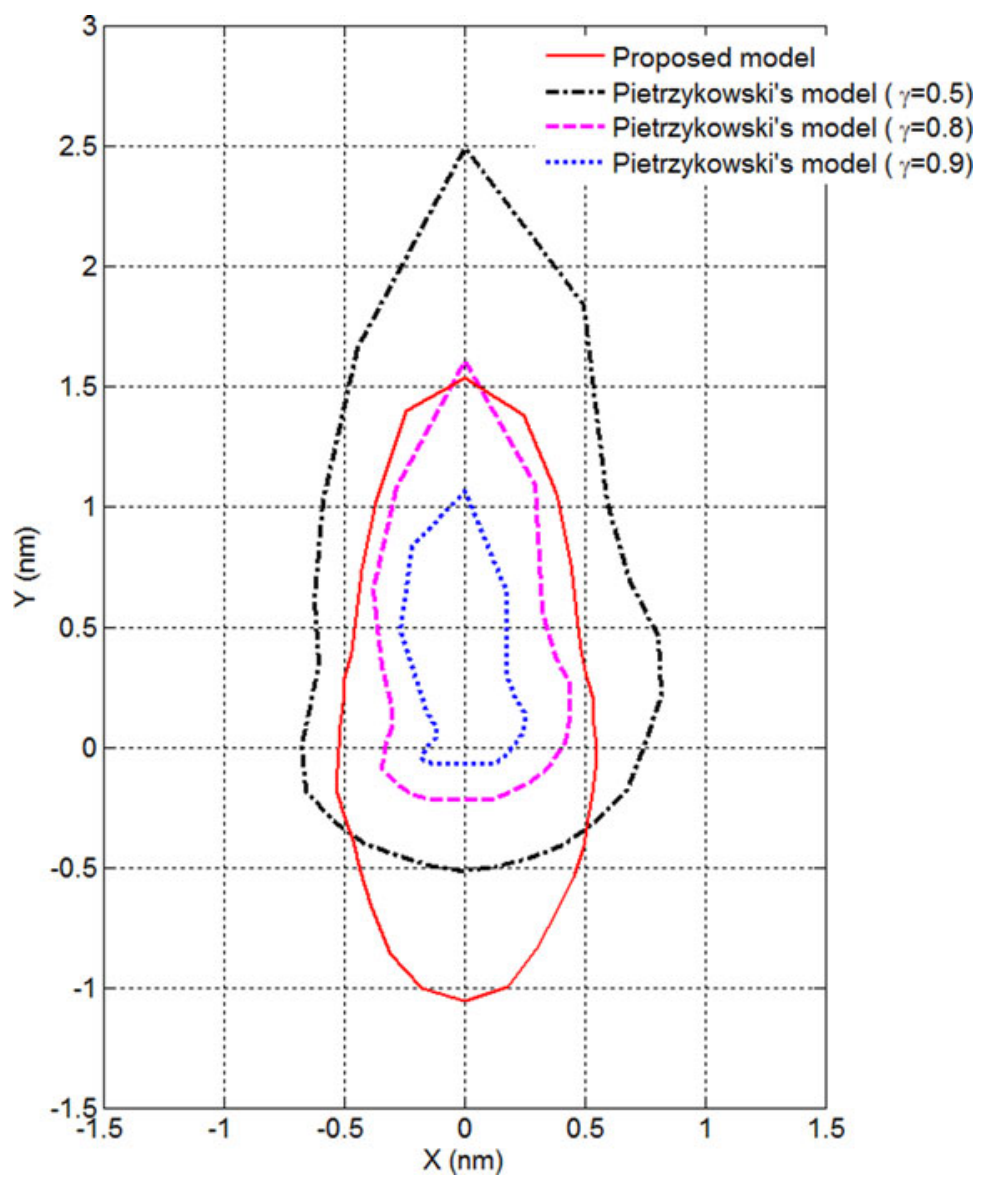

Figure 13. Comparison of safe spacing between proposed model and Pietrzykowski's model in a head-on encounter.

Figure 12 generally have a much larger aft side than the port and starboard sides. This discussion further demonstrates that the proposed model is well developed empirically.

Summarising, there is generally good compatibility between the proposed model and Pietrzykowski's model, suggesting that the consideration of ship length and speed as well as the encounter type in this paper is suitable. In addition, the good match under the more dangerous situation of Pietrzykowski, particularly on the fore side, port side and starboard side provides good justification for the suitability of proposing a domain model that takes into account the encounter type of the ships.

Summarising the foregoing discussions on the comparisons of safe spacing generated by the proposed model with that from the previous models, it may be concluded that on the whole, the proposed model is compatible with other models particularly in the overall shape and on the fore and lateral sides. However, given that the proposed model accounts for different ship sizes and ship speeds as well as the different encounter types between ships, the proposed model that is developed for confined waters can be regarded as superior and more versatile than the models from previous studies. It is 


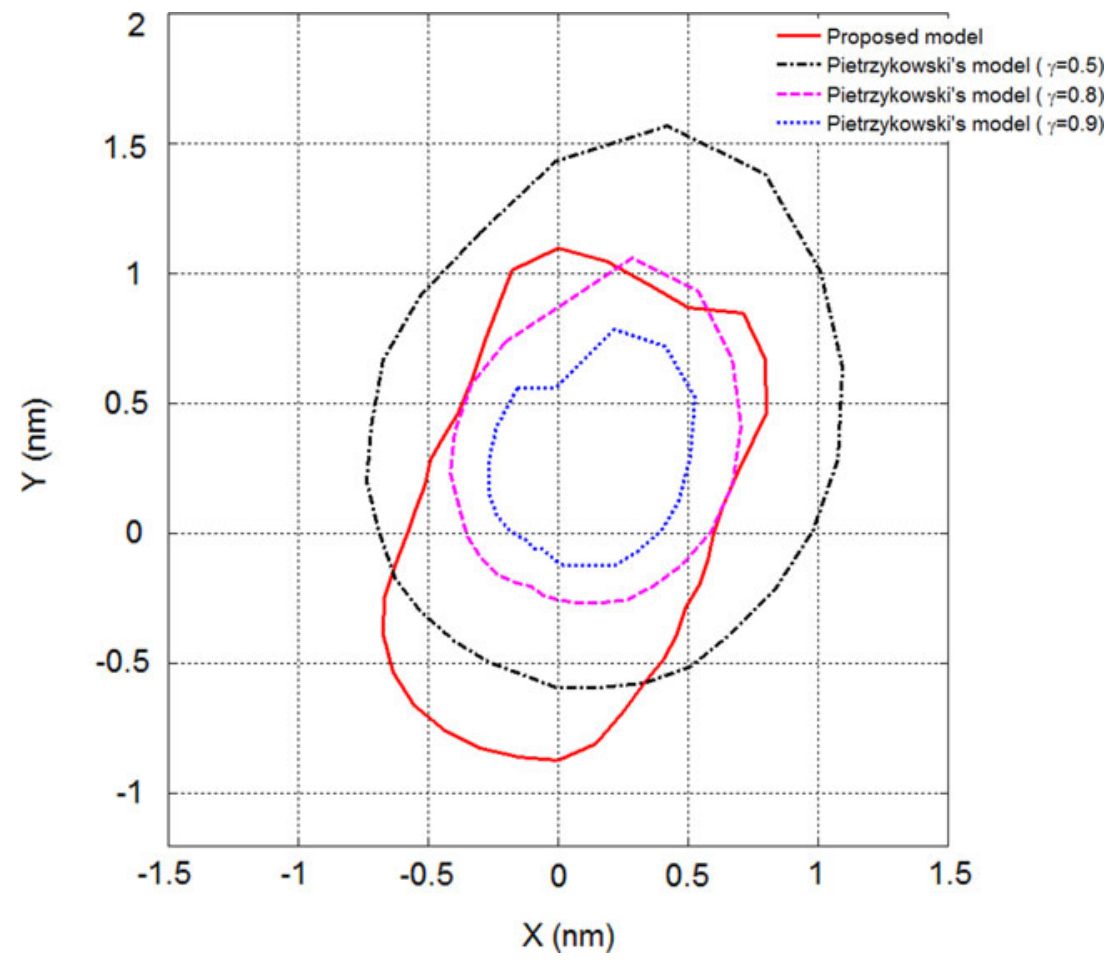

Figure 14. Comparison of safe spacing between proposed model and Pietrzykowski's model in a crossing encounter.

potentially more useful in examining ship separation under dynamically changing encounters and operating conditions.

6. CONCLUSIONS. Literature reviews have shown significant variation in the shape of existing ship domain models. The statistical methods for determining ship domains are also limited due to insufficient dataset, difficulties in separating the factors affecting the domain shape and size as well as unclear definitions. Few ship domain models have been developed for confined waters and the existing poor definitions and modelling of ship encounters may also restrict these empirical ship domains from broader applications. This paper shows that the proposed free-form empirical domain in confined waters can be modelled under a variety of operating conditions and navigational situations. By enforcing two individual domains of an asymmetrical polygonal shape around the OS and TS, the ship domain hence configured is able to provide the required safe distance for any encounter with changing relative bearing and heading. By innovatively employing a weight function, the iterative approach for calibrating the ship domain using vessel movements in Singapore Strait and Singapore Port has demonstrated reasonable results of ship domains. The superiority of the proposed model is demonstrated when compared with existing ship domain models, in that it presents better fitness to the movement dataset compared to Fujii's model and Coldwell's model. 
The methodology used in calibrating the ship domain has great potential in mining trajectory data to study ship navigation. Further work to consider ship trajectories under day and night conditions and the effect of site and channel geometric constraints will allow a further refinement of the ship domain under the influence of other factors.

\section{ACKNOWLEDGEMENTS}

The authors are grateful to the Maritime and Port Authority of Singapore for the data and support in the study. The authors would like to extend special thanks to Capt. Mark Heah for the significant contribution and fruitful suggestion to this paper.

\section{REFERENCES}

Coldwell, T. G. (1983). Marine Traffic Behaviour in Restricted Waters. The Journal of Navigation, 36, 430-444.

COLREGS. (1972). The International Regulations for Preventing Collisions at Sea 1972 (with amendments to 2007 in force from 1 December 2009). International Maritime Organization.

Davis, P. V., Dove, M. J. and Stockel, C. T. (1980). A Computer-Simulation of Marine Traffic Using Domains and Arenas. Journal of Navigation, 33, 215-222.

Dove, M. J., Burns, R. S. and Stockel, C. T. (1986). An Automatic Collision Avoidance and Guidance System for Marine Vehicles in Confined Waters. The Journal of Navigation, 39, 180-190.

Fujii, Y. and Tanaka, K. (1971). Traffic Capacity. Journal of Navigation, 24, 543-552.

Gale, H. and Patraiko, D. (2007). Improving Navigaitonal Safety. Seaways, pp. 4-8.

Goodwin, E. M. (1975). A Statistical Study of Ship Domains. The Journal of Navigation, 28, 328-344.

Hansen, M. G., Jensen, T. K., Lehn-Schiøler, T., Melchild, K., Rasmussen, F. M. and Ennemark, F. (2013). Empirical Ship Domain based on AIS Data. The Journal of Navigation, 66, 931-940.

Kijima, K. and Furukawa, Y. (2003). Automatic Collision Avoidance System Using the Concept of Blocking Area. Proceeding of IFAC Conference on Manoeuvring and Control of Marine Craft, Girona, Spain.

Kijima, K., Furukawa, Y. and Ibaragi, H. (2006). Development of automatic collision avoidance system using the concept of blocking area. Proceeding of 7th IFAC Conference on Manoeuvring and Control of Marine Craft (MCMC), Lisbon, Portugal.

Mathworks. (2012). Global Optimization Toolbox - Solve Multiple Maxima, Multiple Minima, And Nonsmooth Optimization Problems.

Pietrzykowski, Z. (2008). Ship's Fuzzy Domain - a Criterion for Navigational Safety in Narrow Fairways. The Journal of Navigation, 61, 499-514.

Pietrzykowski, Z. and Uriasz, J. (2009). The Ship Domain - A Criterion of Navigational Safety Assessment in an Open Sea Area. Journal of Navigation, 62, 93-108.

Smierzchalski, R. (2000). Ships' Domains as a Collision Risk at Sea in the Evolutionary Trajectory Planning. Proceedings of RISK 2000. Computer Simulation in Risk Analysis and Hazard Mitigation, 11-13 Oct. 2000, Southampton, UK. WIT Press, 43-52.

Smierzchalski, R. and Michalewicz, Z. (2000). Modeling of Ship Trajectory in Collision Situations by an Evolutionary Algorithm. Evolutionary Computation, IEEE Transactions on, 4, 227-241.

Szlapczynski, R. (2011). Evolutionary Sets of Safe Ship Trajectories: a New Approach to Collision Avoidance. The Journal of Navigation, 64, 169-181.

Zhao, J., Tan, M., Price, W. G. and Wilson, P. A. (1994). DCPA Simulation Model for Automatic Collision Avoidance Decision Making Systems Using Fuzzy Sets. Proceedings of OCEANS '94, 'Oceans Engineering for Today's Technology and Tomorrow's Preservation', 2, 244-249.

Zhao, J., Wu, Z. \& Wang, F. (1993). Comments on Ship Domains. The Journal of Navigation, 46, 422-436. 